\title{
Age-specific cut-off levels of anti-Müllerian hormone can be used as diagnostic markers for polycystic ovary syndrome
}

Fahimeh Ramezani Tehrani ${ }^{{ }^{*}}$, Maryam Rahmati ${ }^{1}$, Fatemeh Mahboobifard ${ }^{1}$, Faezeh Firouzi ${ }^{1}$, Nazanin Hashemi ${ }^{1}$ and Fereidoun Azizi ${ }^{2}$

\begin{abstract}
Background: The majority of available studies on the AMH thresholds were not age-specific and performed the receiver operating characteristic curve $(\mathrm{ROC})$ analysis, based on variations in sensitivity and specificity rather than positive and negative predictive values (PPV and NPV, respectively), which are more clinically applicable. Moreover, all of these studies used a pre-specified age categorization to report the age-specific cut-off values of AMH.

Methods: A total of 803 women, including 303 PCOS patients and 500 eumenorrheic non-hirsute control women, were enrolled in the present study. The PCOS group included PCOS women, aged 20-40 years, who were referred to the Reproductive Endocrinology Research Center, Tehran, Iran. The Rotterdam consensus criteria were used for diagnosis of PCOS. The control group was selected among women, aged 20-40 years, who participated in Tehran Lipid and Glucose cohort Study (TLGS). Generalized additive models (GAMs) were used to identify the optimal cutoff points for various age categories. The cut-off levels of $\mathrm{AMH}$ in different age categories were estimated, using the Bayesian method.
\end{abstract}

Main results and the role of chance: Two optimal cut-off levels of AMH $(\mathrm{ng} / \mathrm{ml})$ were identified at the age of 27 and 35 years, based on GAMs. The cut-off levels for the prediction of PCOS in the age categories of 20-27, 27-35, and 35-40 years were 5.7 (95\% Cl: 5.48-6.19), 4.55 (95\% Cl: 4.52-4.64), and 3.72 (95\% Cl: 3.55-3.80), respectively. Based on the Bayesian method, the PPV and NPV of these cut-off levels were as follows: PPV $=0.98$ (95\% Cl: $0.96-$ $0.99)$ and NPV $=0.40$ (95\% Cl: 0.30-0.51) for the age group of 20-27 years; PPV $=0.96$ (95\% Cl: $0.91-0.99)$ and $\mathrm{NPV}=0.82$ (95\% Cl: 0.78-0.86) for the age group of $27-35$ years; and PPV $=0.86$ (95\% Cl: 0.80-0.94) and NPV $=0.96$ (95\% Cl: 0.93-0.98) for the age group of 35-40 years.

Conclusions: Application of age-specific cut-off levels of AMH, according to the GAMs and Bayesian method, could elegantly assess the value of AMH in discriminating PCOS patients in all age categories.

Keywords: Anti-Müllerian hormone, Polycystic ovary syndrome, Age-specific, Diagnosis, Bayesian method

\footnotetext{
*Correspondence: ramezani@endocrine.ac.ir; fah.tehrani@gmail.com; framezan@post.harvard.edu

${ }^{1}$ Reproductive Endocrinology Research Center, Research Institute for Endocrine Sciences, Shahid Beheshti University of Medical Sciences, P.O.

Box:19395-4763, 24 Parvaneh, Yaman Street, Velenjak, I.R 1985717413 Tehran, Iran

Full list of author information is available at the end of the article
}

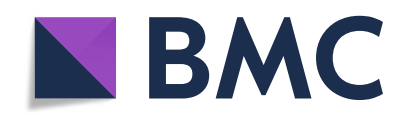

(- The Author(s). 2021 Open Access This article is licensed under a Creative Commons Attribution 4.0 International License, which permits use, sharing, adaptation, distribution and reproduction in any medium or format, as long as you give appropriate credit to the original author(s) and the source, provide a link to the Creative Commons licence, and indicate if changes were made. The images or other third party material in this article are included in the article's Creative Commons licence, unless indicated otherwise in a credit line to the material. If material is not included in the article's Creative Commons licence and your intended use is not permitted by statutory regulation or exceeds the permitted use, you will need to obtain permission directly from the copyright holder. To view a copy of this licence, visit http://creativecommons.org/licenses/by/4.0/ The Creative Commons Public Domain Dedication waiver (http://creativecommons.org/publicdomain/zero/1.0/) applies to the data made available in this article, unless otherwise stated in a credit line to the data. 


\section{Background}

Polycystic ovary syndrome (PCOS) is the most common endocrine disorder in reproductive women. The prevalence of PCOS ranges from 5 to $20 \%$ in different studies, depending on the recruitment process of the study population, the criteria used for its definition, and the method used to define each criterion [1]. According to the Rotterdam criteria, which were first introduced in 2004, diagnosis of PCOS requires the presence of at least two of the following findings: (i) oligoanovulation (OA); (ii) clinical or biochemical hyperandrogenism (HA); and (iii) polycystic ovarian morphology (PCOM), based on the ultrasound findings. Also, other etiologies for excess androgen production must be excluded [2]. However, the inclusion of PCOM in the definition of PCOS has caused major concerns regarding the validity and reliability of its assessment and highlighted the need for revising its definition, given the application of advanced high-resolution ultrasound devices [3].

Several efforts have been made to introduce a more reliable alternative for PCOM. The anti-Müllerian hormone (AMH), given its exclusive production by granulosa cells of the ovary, has been shown to be the best marker, reflecting the antral follicle count (AFC) [4]. Researchers have also attempted to identify the optimal diagnostic threshold for AMH to precisely identify PCOM [5] for the PCOS criteria. However, there is no universally accepted threshold [5]. Besides AFC, AMH is correlated with HA [6-8], oligomenorrhea, and menstrual disorders [9].

Several studies have suggested that AMH could be used as a surrogate marker for the diagnosis of PCOS [10-12]. However, the majority of these studies have some shortcomings, mainly due to not being agespecific, as marked changes occur in AMH across the reproductive lifespan in the normal population, and there is the possibility of AMH decline in a less rapid manner in women with PCOS $[13,14]$. Therefore, in the present study, we aimed to introduce the age-specific cut-off levels of AMH for the prediction of PCOS, using a Bayesian method, and to identify the optimal cut-off points for various age categories, using generalized additive models (GAMs).

\section{Methods}

This study was conducted on 839 women at reproductive age, including 321 PCOS patients (case group) and 518 eumenorrheic non-hirsute women (control group), aged 20-40 years. The controls were selected among the participants of Tehran Lipid and Glucose cohort Study (TLGS), which is a cohort study among a representative sample of residents in Tehran, Iran. Face-to-face interviews were conducted with 1060 women, aged 18-45 years. The reproductive history, with emphasis on the menstrual cycle, gynecological history, hyperandrogenic symptoms, and family history of irregular menstrual cycle and hirsutism were collected. Hirsutism was assessed by a trained general practitioner, under the supervision of a single gynecologist, using the modified Ferriman-Gallwey (mFG) scoring method. For cases with acne and/or an initial mFG score $>3$ and/or a menstrual disorder (defined as a menstrual interval $<21$ days or $>$ 35 days), the androgen and/or progesterone profile in the mid-luteal phase was assessed. The details are published elsewhere [15]. To select the controls, we excluded women with at least one of the PCOS criteria, including irregular or unpredictable menstrual cycles, subclinical anovulation (progesterone level $<4 \mathrm{ng} / \mathrm{mL}$ in two consecutive cycles), and biochemical and/or clinical HA $(n=307)$. In addition, the exclusion criteria were as follows: (1) menopause $(n=22)$; (2) history of hysterectomy, oophorectomy, or ovarian surgeries $(n=36)$; (3) history of endocrine disorders or use of medications that could affect the function of the hypothalamic-pituitarygonadal (HPG) axis $(n=34)$; (4) lack of available information on the reproductive history $(n=60)$; (5) being in the age range of $<20$ or $>40$ years $(n=83)$; and (6) having an outlier AMH value $(n=18)$.

The PCOS group consisted of PCOS women, aged 20-40 years, who were referred to the Reproductive Endocrinology Research Center, Tehran, Iran. The Rotterdam criteria were used for diagnosis of PCOS, based on the presence of at least two of the three following findings: (i) OA; (ii) HA; and (iii) PCOM on ultrasound. OA was considered as vaginal bleeding episodes at no less than 35-day intervals or progesterone levels $<4 \mathrm{ng} /$ $\mathrm{mL}$ in two consecutive cycles. Also, HA was defined as clinical hyperandrogenism, that is, the presence of hirsutism ( $\mathrm{mFG} \geq 8$ ), acne, or androgenic alopecia and/or biochemical hyperandrogenism (BH).

$\mathrm{BH}$ was detected based on the free androgen index (FAI) and the level of dehydroepiandrosterone sulfate (DHEAS) and/or androstenedione (A4) above the upper 95th percentile in women $(n=362)$, who were not using any hormonal medications and had no clinical evidence of HA, OA, or PCOM. Specifically, the upper normal limits were as follows: DHEAS $=246 \mu \mathrm{g} / \mathrm{dL}, \mathrm{FAI}=5.47$, total testosterone $=0.88 \mathrm{ng} / \mathrm{mL}, \quad \mathrm{A} 4=2.3 \mathrm{ng} / \mathrm{mL}$ [16]. PCOM was diagnosed, based on the presence of $\geq 12$ follicles $2-9 \mathrm{~mm}$ in each ovary and/or increased ovarian volume $\left(10 \mathrm{~cm}^{3}\right)$. Out of 321 women with PCOS, those with an outlier AMH value were excluded $(n=18)$.

An overnight fasting venous blood sample was obtained from each participant on the second or third day of their spontaneous or progesterone-induced menstrual cycle. The samples were centrifuged within $30-45 \mathrm{~min}$ of collection and stored at $-80{ }^{\circ} \mathrm{C}$ until further analysis. All AMH measurements were performed by an 
experienced laboratory technician in the same laboratory at the Research Institute for Endocrine Sciences, using the same assay method and kit (Gen II Kit, Beckman Coulter, Inc., Fullerton, California, USA), as well as the same Sunrise ELISA reader (Tecan Co., Salzburg, Austria). Also, the AMH Gen II control (A79766) was used at two concentrations to monitor the accuracy of the assays. The intra- and inter-assay coefficients of variation were 1.9 and $2.0 \%$, respectively.

\section{Statistical analysis}

Data are presented as mean \pm standard deviation (SD) for numerical variables with normal distributions, as median and interquartile range (IQR) for variables with skewed distributions, and as number (percentage) for categorical variables. The characteristics of women with PCOS and the controls were compared, using student's t-test for normally distributed continuous variables and Chi-square test for categorical data. Mann-Whitney U test was also performed to compare variables with skewed distributions.

To measure the outliers of AMH, Tukey's test was performed, as it makes no distributional assumptions. Accordingly, all values, which were more than 1.5 times the IQR below the first quartile or above the third quartile (either below Q1 - 1.5 IQR or above Q3 + 1.5 IQR), were excluded. To detect the correlation of AMH with age and body mass index (BMI), a scatter plot matrix was drawn, and a fractional polynomial (FP) regression model was fitted to identify the non-linear relationship between continuous variables (age and AMH level). Pearson's or Spearman's correlation test was also used to examine the correlation of AMH with age and BMI.

It seems that subjective categorization of age (i.e., 20$25,25-30,30-35$, and $35-40$ years) is not appropriate for age-specific predictions; therefore, GAMs were used to identify the cut-off points for age, considering the association of this variable with AMH [17]. These models consider non-parametric functions of predictive variables (i.e., age and AMH), which are associated with the dependent variable (i.e., PCOS) using a logit link function and provide the optimal cut-off points, based on the area under the ROC curve (AUC) maximization [18].

Next, the cut-off values of AMH were estimated, based on the negative and positive predictive values (NPV and PPV, respectively) for different age categories, using the Bayesian method, introduced by Vradi et al. [19]. Generally, this method models the probability of PCOS with a step function, based on the predictive values, and estimates the cut-off value, as well as the predictive values. Here, the cut-off value was a parameter of the model, and therefore, a Bayesian method could be applied. This method allows direct probability interpretations of parameters, based on the observed data and uses both prior and sample information [20]. Moreover, the predictive summary index (PSI) was applied, which is a frequentist approach to determine the optimal overall and age-specific cut-off levels of AMH. It estimates the optimal cut-off value by maximizing the difference in predictive values for all possible cut-off points and is expressed as follows [19]:

$$
P S I=\max _{\text {cutoff }}\left\{P P V_{\text {cutoff }}+N P V_{\text {cutoff }}-1\right\}
$$

For the latter approach, the confidence intervals were calculated by the bootstrap method.

There was not an adequate sample size to calculate the age-specific cut-off values of AMH for different PCOS phenotypes; however, these cut-offs for different phenotypes (regardless of age) were estimated, using the proposed Bayesian method.

Data were analyzed using OpenBUGS version 3.2.3 and $\mathrm{R}$ software version 3.6.1. The optimal cut-off points for age were obtained by the CatPredi package in $\mathrm{R}$ software. $P$-value less than 0.05 was considered statistically significant.

\section{Results}

Of a total of 839 subjects, aged $20-40$ years, $321(38.2 \%)$ women had PCOS, and 518 (61.8\%) were normoovulatory. The participants with outlier AMH levels were detected and excluded, based on the boxplot method. Finally, 803 subjects, including $303(37.7 \%)$ patients with PCOS and $500(62.3 \%)$ normo-ovulatory women, were examined. Women in the PCOS group were younger

Table 1 Clinical and endocrine characteristics of the PCOS cases and normo-ovulatory controls

\begin{tabular}{|c|c|c|c|}
\hline Variable & $\begin{array}{l}\text { Normo-ovulatory } \\
(n=500)\end{array}$ & $\begin{array}{l}\text { PCOS } \\
(303)\end{array}$ & $p$-value \\
\hline Age (years) & $33.1 \pm 4.6$ & $27.9 \pm 4.6$ & $<0.001^{a}$ \\
\hline Age at menarche (years) & $13.5 \pm 1.4$ & $13.1 \pm 1.6$ & $0.002^{a}$ \\
\hline Number of pregnancy & $2.5 \pm 1.2$ & $0.4 \pm 0.8$ & $<0.001^{a}$ \\
\hline Number of delivery & $2.1 \pm 0.9$ & $0.3 \pm 0.6$ & $<0.001^{a}$ \\
\hline Number of abortion & $1.2 \pm 0.6$ & $0.1 \pm 0.5$ & $<0.001^{a}$ \\
\hline BMI $\left(\mathrm{kg} / \mathrm{m}^{2}\right)$ & $26.5 \pm 4.5$ & $26.7 \pm 5.7$ & 0.5 \\
\hline WC $(\mathrm{cm})$ & $83.5 \pm 10.3$ & $85.5 \pm 12.8$ & $0.01^{a}$ \\
\hline Wrist (cm) & $15.7 \pm 0.9$ & $15.3 \pm 1.2$ & $<0.001^{a}$ \\
\hline Hip (cm) & $103.2 \pm 8.8$ & $104.4 \pm 10.6$ & 0.08 \\
\hline WHR & $0.8 \pm 0.1$ & $0.82 \pm 0.1$ & 0.9 \\
\hline WHtR & $0.5 \pm 0.1$ & $0.5 \pm 0.1$ & 0.8 \\
\hline $\mathrm{AMH}(\mathrm{ng} / \mathrm{ml})^{b}$ & $1.70(0.85-2.81)$ & $6.21(3.90-9.03)$ & $<0.001^{a}$ \\
\hline
\end{tabular}

Note: Data are presented as mean \pm standard deviation unless otherwise indicated. Independent t-test, or Mann-Whitney test were used as appropriate $B M I$ body mass index, $A M H$ anti-mullerian hormone, WC waist circumference, WHR waist-to-hip ratio, WHtR waist-to-height ratio

${ }^{\text {aS }}$ Statistically significant result $(p<0.05)$

${ }^{\mathrm{b}}$ Median (IQR 25-75\%) 
(27.9 \pm 4.6 vs. $33.1 \pm 4.6)$ than the control group and had significantly higher AMH levels (6.21, IQR: 3.90-9.03 vs. 1.70, IQR: $0.85-2.81 ; P<0.001)$. There was no significant difference in terms of BMI between the PCOS and control groups $(P=0.5)$. The clinical and endocrine characteristics of the PCOS group and normo-ovulatory controls are summarized in Table 1.

As shown in Fig. 1, the scatter plot matrix indicated that $\mathrm{AMH}$ levels decreased with increasing age in both PCOS and normo-ovulatory groups $\left(\mathrm{r}_{\text {total }}=\right.$ $-0.63, P<0.001)$; however, there was no significant association between the AMH level and $\mathrm{BMI}\left(\mathrm{r}_{\text {total }}=\right.$
-0.05, $P=0.12$ ). The cut-off points for age were obtained, based on the GAM method, where the predictive variables (i.e., age and $\mathrm{AMH}$ ) were included in the logit link function. Two optimal cut-off points were identified at the age of 27 and 35 years, and the age groups were classified as follows: 20-27, 27-35, and 35-40 years (Fig. 2). The distribution of AMH in the PCOS and control groups, based on age stratification, is presented in Fig. 3. The AMH values were compared between the PCOS and normo-ovulatory control groups in an age-stratified analysis. The level of AMH decreased with increasing age, and a
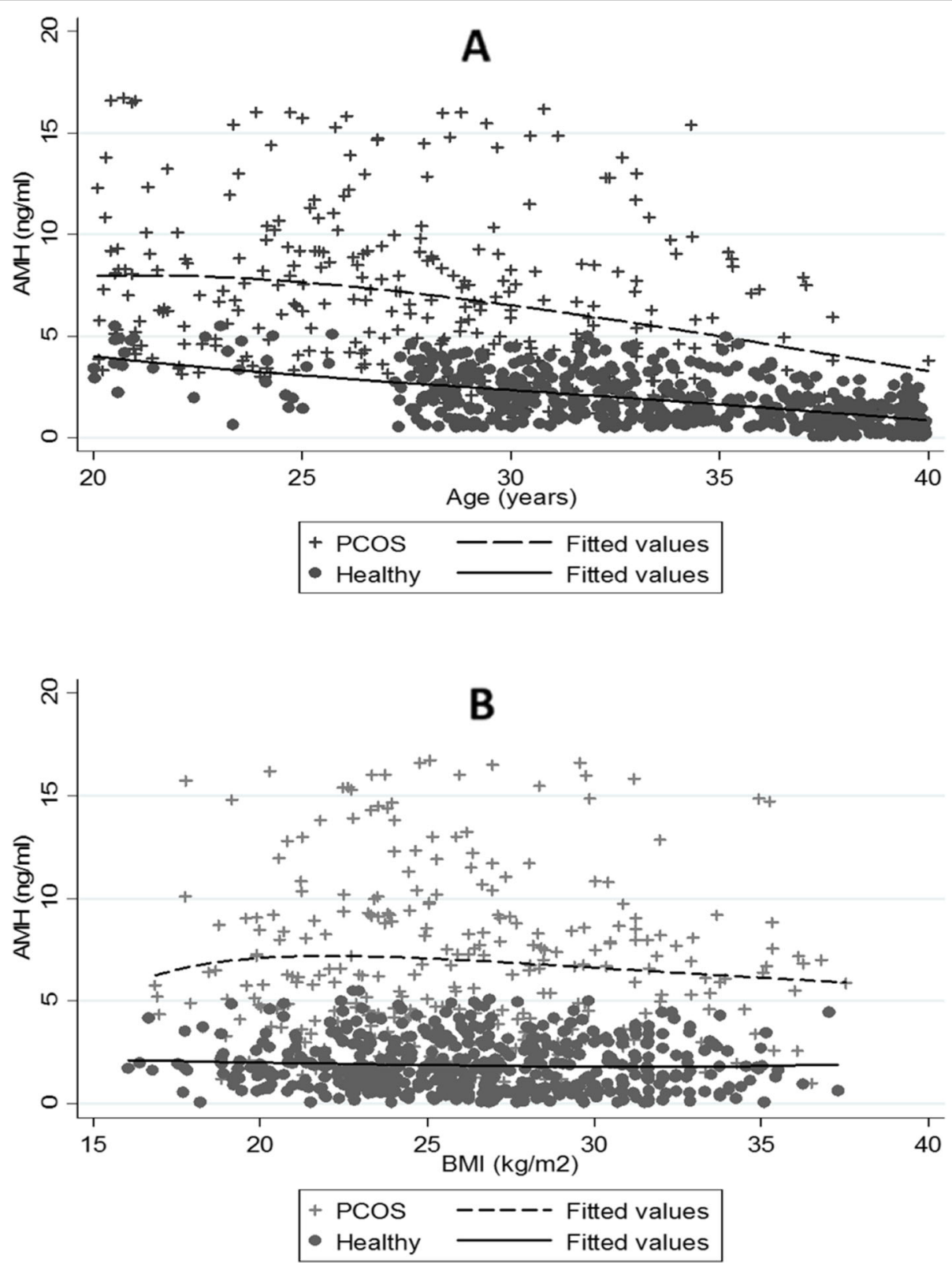

Fig. 1 Scatter plot matrix of AMH levels versus (a) age and (b) BMI 


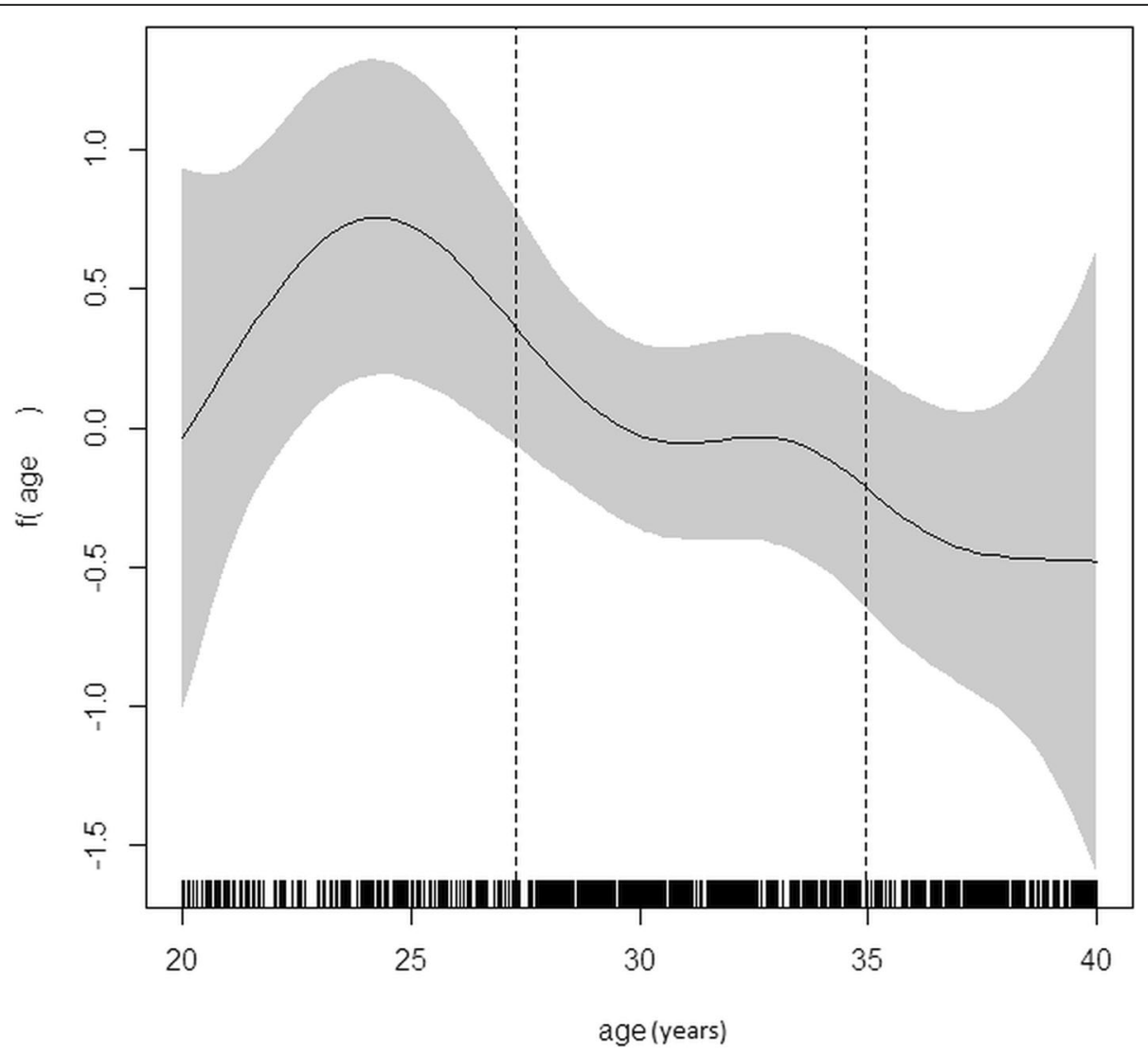

Fig. 2 The optimal location of cut points for age variable based on the generalized additive models (GAMs)

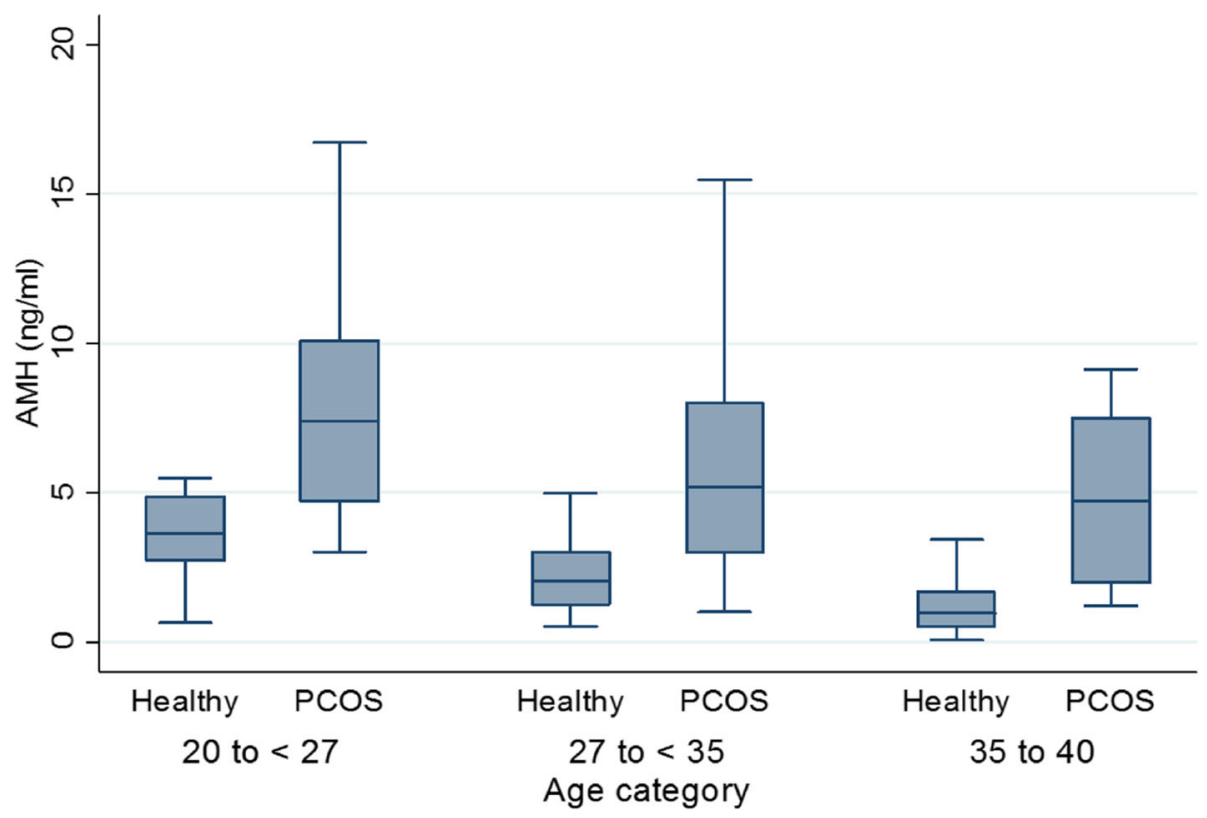

Fig. 3 Box plots showing the values of serum AMH in $\mathrm{ng} / \mathrm{ml}$ within controls and PCOS and with stratification by age group 
Table 2 Median and (IQR 25-75\%) for AMH (ng/ml) variable by age categories in PCOS and normo-ovulatory

\begin{tabular}{llll}
\hline Age category $^{\mathbf{a}}$ & $\begin{array}{l}\text { Normo-ovulatory } \\
(\boldsymbol{n}=\mathbf{5 0 0})\end{array}$ & $\begin{array}{l}\text { PCOS } \\
(\boldsymbol{n}=\mathbf{3 0 3})\end{array}$ & $\boldsymbol{p}$-value \\
\hline $\mathbf{2 0}$ to $<\mathbf{2 7}$ & $N=30$ & $N=134$ & $<0.001$ \\
& $3.6(2.60-4.85)$ & $7.4(4.70-10.12)$ & \\
$\mathbf{2 7}$ to $<\mathbf{3 5}$ & $N=284$ & $N=151$ & $<0.001$ \\
& $2.03(1.23-3.02)$ & $5.16(2.97-8.00)$ & \\
$\mathbf{3 5}$ to $\mathbf{4 0}$ & $N=186$ & $N=18$ & $<0.001$ \\
& $0.97(0.48-1.68)$ & $4.72(1.89-7.60)$ & \\
\hline
\end{tabular}

${ }^{\mathrm{a}}$ Age categories were determined using the generalized additive models (GAMs)

significant difference was found between the PCOS and normo-ovulatory groups $(P<0.001)$ (Table 2$)$.

Figure 4 presents the posterior distribution of the cutoff values of AMH for PCOS diagnosis in the aforementioned age categories and the total population. The posterior mean of the cut-off AMH values in the age groups was as follows: 5.7 (95\% CI: 5.48-6.19), 4.55 (95\% CI: 4.52-4.64), and 3.72 (95\% CI: 3.55-3.80) for the age groups of 20-27, 27-35, and 35-40 years, respectively.
At these cut-off levels, the Bayesian posterior mean values, based on PPV and NPV, were as follows: PPV = 0.98 (95\% CI: $0.96-0.99)$ and NPV $=0.40$ (95\% CI: $0.30-0.51$ ) for the age group of 20-27 years; $\mathrm{PPV}=0.96$ (95\% CI: 0.91-0.99) and NPV $=0.82$ (95\% CI: $0.78-$ 0.86 ) for the age group of $27-35$ years; and $\mathrm{PPV}=0.86$ (95\% CI: $0.80-0.94)$ and NPV $=0.96$ (95\% CI: $0.93-$ 0.98 ) for the age group of 35-40 years (Table 3). Regardless of the age categories, the posterior mean of the cutoff AMH value was 4.54 (95\% CI: 4.18-5.12), with PPV of 0.93 (95\% CI: $0.89-0.99)$ and NPV of 0.83 (95\% CI: 0.79-0.87). The boxplot and the cut-off values of AMH within PCOS phenotypes, (regardless of age specification due to the lack of enough sample size), are presented in supplementary Fig. 1 and supplementary Table 1. Overall, the posterior means of the cut-off AMH values for the PCOS phenotypes were in a similar range.

The optimal overall and age-specific cut-off values for $\mathrm{AMH}$, based on the PSI method, are presented in Table 3. The cut-off $A M H$ value in the total population was 4.60 (95\% CI: 4.13-5.54), with PPV of 0.93 (95\% CI: 0.89-0.96) and NPV of 0.83 (95\% CI: 0.79-0.86). Also, the cut-off values of AMH for the age categories of 20

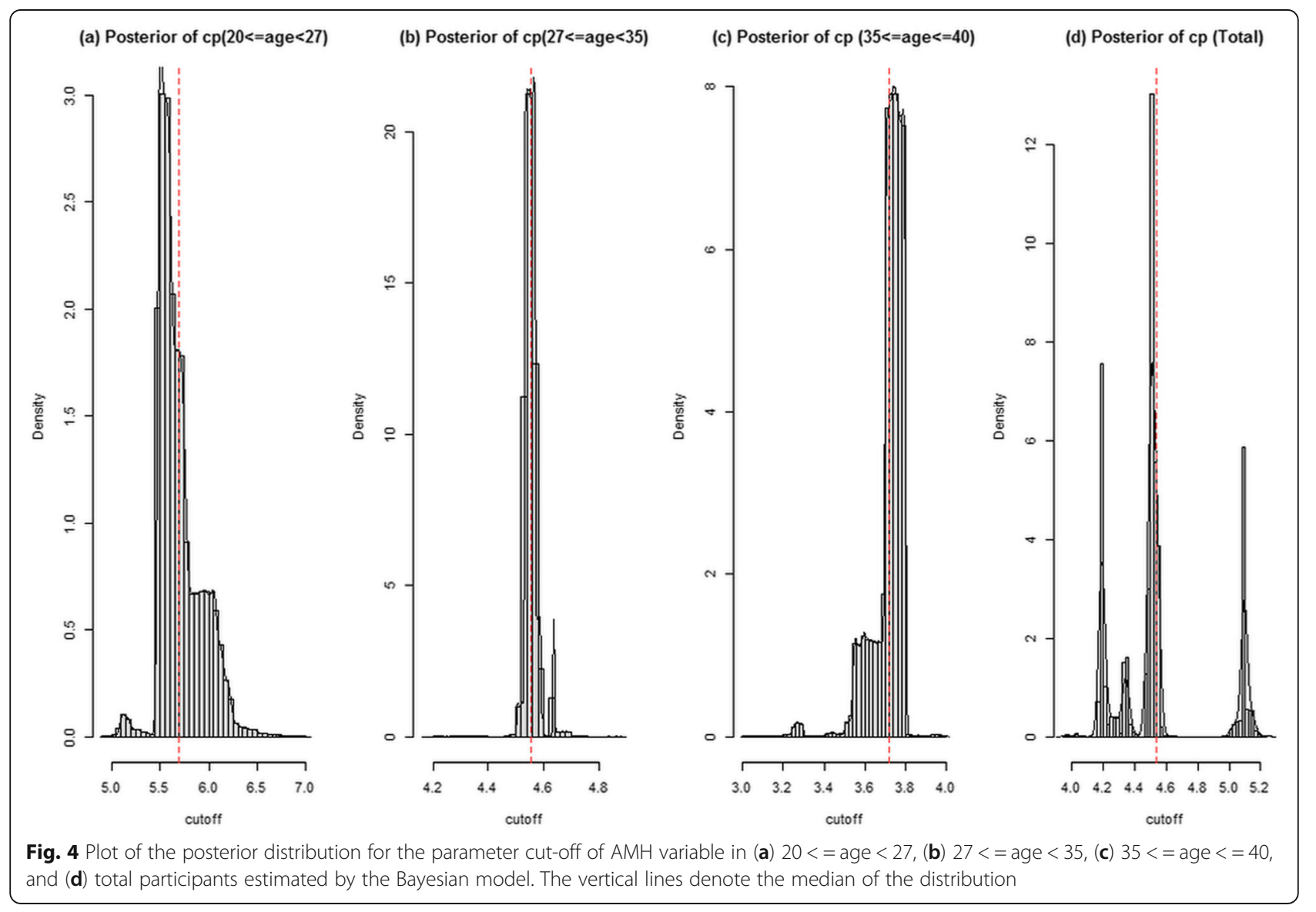


Table 3 Cut-off, PPV and NPV for AMH values (ng/ml), based on Bayesian approach and PSI method with 95\% Cl

\begin{tabular}{lllll}
\hline & Age category $^{\mathbf{a}}$ & Estimated cutoff & PPV & NPV \\
\hline Bayesian method & 20 to $<27$ & $5.70(5.48,6.19)$ & $0.98(0.96,0.99)$ & $0.40(0.30,0.51)$ \\
& 27 to $<35$ & $4.55(4.52,4.64)$ & $0.96(0.91,0.99)$ & $0.82(0.78,0.86)$ \\
& 35 to 40 & $3.72(3.55,3.80)$ & $0.86(0.80,0.94)$ & $0.96(0.93,0.98)$ \\
PSI method & Total & $4.54(4.18,5.12)$ & $0.93(0.89,0.99)$ & $0.83(0.79,0.87)$ \\
& 20 to $<27$ & $8.51(7.12,9.38)$ & $0.85(0.81,0.89)$ & $0.48(0.25,0.72)$ \\
& 27 to $<35$ & $2.91(2.11,3.72)$ & $0.65(0.61,0.69)$ & $0.92(0.89,0.95)$ \\
& 35 to 40 & $1.88(1.25,2.21)$ & $0.57(0.57,0.61)$ & $0.94(0.91,0.97)$ \\
& Total & $4.60(4.13,5.54)$ & $0.93(0.89,0.96)$ & $0.83(0.79,0.86)$ \\
\hline
\end{tabular}

Note: PPV Positive predictive value, NPV Negative predictive value, PSI predictive summary index, Cl confidence interval

${ }^{a}$ Age categories were determined using the generalized additive models (GAMs)

27, 27-35, and 35-40 years were 8.51 (95\% CI: 7.129.38), 2.91 (95 \% CI: 2.11-3.72), and 1.88 (95\% CI: $1.25-$ $2.21)$, respectively.

We also considered several simulation scenarios with different sample sizes to compare two methods (Bayesian and PSI). In addition, three different prior specifications, including uninformative, informative and mixture priors were considered. The results showed that the Bayesian method gives much better coverage than the PSI method for a small sample size. Also, for all considered priors, the resulting estimators are on average unbiased. For the PSI method in a small sample size (under 50 ), the bias of the estimate of the cut point is far too high in absolute terms. The length of the credible interval for the Bayesian method was always narrower compared to PSI (data not shown).

\section{Discussion}

In the present study, which was conducted on a large population of women diagnosed with PCOS (based on the Rotterdam criteria) and eumenorrheic non-hirsute controls, we reported age-specific cut-off values of AMH for the prediction of PCOS, using the Bayesian method. This method is based on reporting PPV and NPV, which demonstrate more clinical utility for clinicians, compared to the ROC analysis, indicating sensitivity and specificity [19]. Additionally, this method has the advantage of dealing with a small sample size because of not relying on asymptotic theory $[21,22]$. A comparison of the Bayesian method with the PSI index (as an alternative frequentist method) in our data set, revealed that the Bayesian method provides more informative results. Moreover, we categorized age, based on GAM models, which provide more reliable cut-off points than subjective categorization of age. Based on these methods, an AMH cut-off value of $5.7 \mathrm{ng} / \mathrm{mL}$ was obtained for women, aged 20-27 years, with PPV of 0.98 (95\% CI: 0.96-0.99). The AMH cut-off value was $4.55 \mathrm{ng} / \mathrm{mL}$ for women aged $27-35$ years and 3.72 for those aged $35-40$ years, with PPVs of 0.96 (95\% CI: 0.91-0.99) and 0.86 (95 \% CI: 0.80-0.94), respectively.

Considering the limitations of the Rotterdam criteria, including ultrasound challenges (e.g., lack of universally approved cut-off points for exploring the follicle count to define PCOM, limited availability of ultrasound devices, and high cost) [5] and regional variations in the clinical presentations of HA [23, 24], use of a single assay to diagnose PCOS has been encouraged. Among candidate markers, AMH seems to be an eligible candidate. The circulating level of $\mathrm{AMH}$ is not majorly influenced by the menstrual cycle or use of contraceptives [25]. It also shows a strong association with AFC and serves as a reliable marker for the ovarian reserve [26].

$\mathrm{AMH}$ has been shown to be a proper alternative for the follicle count on ultrasound images in the Rotterdam criteria [10, 27], considering its superior AUC and higher sensitivity and specificity than the follicle count [3]. This marker can also discriminate between PCOM and PCOS $[28,29,5,30]$. AMH has been proposed as an effective endocrine factor in the pathophysiology of PCOS, considering its elevated serum level in PCOS, its overproduction by granulosa cells in anovulatory PCOS, its cross-communication with luteinizing hormone $(\mathrm{LH})$ and follicle-stimulating hormone (FSH) leading to HA, and finally, its correlation with the severity of ovulatory dysfunction and PCOS [28, 31].

It is well established that follicle count per ovary and ovarian volume show decreasing trends with age. The use of these markers, by establishing age-specific thresholds, has provided higher sensitivity and specificity for diagnosis of PCOS, compared to a single threshold [32]. Moreover, studies have shown that AMH levels gradually decrease with aging [13]; however, the rate of $\mathrm{AMH}$ decline may not be the same for all women at reproductive age [14]. It has been shown that the ovarian pool is depleted in a more gradual manner in the ovaries of PCOS women, compared to non-PCOS women [33].

Evidence shows that the AMH reduction rate accelerates after the age of 40 years [34]. However, this decline 
has been found to be slower over time in PCOS women than normo-ovulatory women, indicating sustained fertility in PCOS women [33]. Several efforts have been made to provide an optimal single threshold of AMH for the precise diagnosis of PCOS. According to a metaanalysis of ten studies, as well as a study utilizing three main criteria (NIH, Rotterdam, and AE-PCOS criteria) to distinguish PCOS $[35,36]$, the AMH cut-off value of $\sim 4.7 \mathrm{ng} / \mathrm{mL}$ was the best single threshold to distinguish PCOS women, based on the Rotterdam criteria. However, the majority of these studies did not report agespecific thresholds [35] and used ROC analysis, which represents sensitivity and specificity rather than PPV and NPV.

In the present study, we used the Bayesian approach [19]. Besides providing PPV and NPV values, this method allows us to consider a posterior probability distribution, according to previously reported thresholds to improve the estimations. Moreover, age categories have been subjectively identified in all previous studies, while we used GAMs with a logit link function to obtain the optimal cut-off points for age, based on AUC maximization [18, 37]. Generally, GAMs consider the nonlinear dependence between the covariates and the expected value of the response variable and provide more flexibility than generalized linear models by considering the non-parametric functions of predictive variables [17].

Besides the Bayesian method, we also reported the optimal overall and age-specific cut-off values for AMH with an alternative approach, that is, PSI. Similar to the Bayesian method, PSI is based on reporting PPV and NPV. However, it does not directly provide confidence intervals for the parameters of interest. PSI is indirectly calculated, using bootstrap samples. In the present study, the optimal single thresholds of $\mathrm{AMH}$, calculated with both Bayesian and PSI methods, were 4.54 and 4.60, respectively. These thresholds could noticeably discriminate PCOS women $(\mathrm{PPV}=0.93)$ from non-PCOS women $(\mathrm{NPV}=0.83)$. After stratifying the AMH values by age, it was found that PPV for the younger age categories (2027 and 27-35 years) and NPV for the oldest age category (35-40 years) were improved. Overall, the PSI approach showed wider confidence intervals and lower PPVs, compared to the Bayesian method.

There is no consistence regarding the association between BMI and PCOS or between BMI and AMH [36, $38,39]$. In this regard, a meta-analysis conducted by Moslehi et al. [40] revealed that markers of ovarian reserve (AMH and FSH) were significantly lower in obese women, compared to non-obese women. Also, BMI was negatively correlated with $\mathrm{AMH}$ in all populations and with FSH in fertile non-PCOS subgroups [40]. It seems that the association between $\mathrm{AMH}$ and PCOS is affected by BMI $[31,38]$. In the present study, there was no significant association between AMH and BMI in either of the groups, and no significant difference was found in terms of BMI between PCOS and control women. Therefore, we did not adjust the age-specific AMH cutoff values for BMI.

The present study, which included community controls, benefitted from the Bayesian method to determine the cut-off AMH values for different age groups, identified by GAMs. This method provides PPVs and NPVs, which are more useful for clinical decision-making. Moreover despite the limitations of the small sample size of 30 cases in controls (20-27 years) and 18 in PCOS (35-40 years), the Bayesian method was very tractable in estimating the parameters of interest, resulting in point estimators (e.g. posterior mean) that are practically unbiased in all scenarios, for all prior constellations and sample size assumptions; this issue is in line with recommendations to use Bayesian over frequentist estimation in small sample sizes [41, 42]. Also, the intra-assay and inter-assay variabilities of AMH measurements seem to be ignored, since all $\mathrm{AMH}$ assays were performed by an expert in the same laboratory. Moreover, the participants in the current study were in wider age groups, compared to previous studies; this enabled us to provide thresholds for all reproductive age groups. On the other hand, our study had some limitations. First, due to the decline in PCOS clinical manifestations, concerns and consequences over the lifespan [43-45], the PCOS population is significantly younger than control subjects in our study. Second, we did not use the most sensitive assay for AMH measurements (picoAMH assay). However, the results of Gen II and picoAMH assays are highly correlated and can be translated using an equation (picoAMH $=0.01+1.69 *$ GenII) $\quad[46,47]$. Third, some factors, including variations in AMH levels across the lifespan, the existence of overlap in AMH values between PCOS and healthy subjects with good ovarian reserve, may make universal acceptance of cut-off $\mathrm{AMH}$ levels for the prediction of PCOS be challenging.

\section{Conclusions}

In conclusion, age-specific cut-off values of AMH, using well-established advanced statistical methods could elegantly assess the value of AMH in discriminating PCOS patients and may be useful as an initial assay for PCOS diagnosis.

\section{Abbreviations}

AMH: Anti-Müllerian hormone; PCOS: Polycystic ovary syndrome; PPV: Positive predictive value; NPV: Negative predictive value; TLGS: Tehran Lipid and Glucose cohort Study; GAMs: Generalized additive models; OA: Oligoanovulation; HA: Hyperandrogenism; PCOM: Polycystic ovarian morphology; AFC: Antral follicle count; mFG: Modified Ferriman-Gallwey; DHEAS: Dehydroepiandrosterone sulfate; LH: Luteinizing hormone; FSH: Follicle-stimulating hormone 


\section{Supplementary Information}

The online version contains supplementary material available at https://doi. org/10.1186/s12958-021-00755-8.

Additional file 1: Supplementary Figure 1. Box plots showing the median (Inter Quartile Range) values of serum AMH in $\mathrm{ng} / \mathrm{ml}$ within PCOS phenotypes. Supplementary Table 1. Cutoff, PPV and NPV based on Bayesian approach with 95\% Cl for PCOS phenotypes.

\section{Acknowledgements}

The authors wish to acknowledge the study participants, the staff at the Tehran Lipid and Glucose Study (TLGS) Unit and personnel of the Research Institute for Endocrine Science. The authors also thank Dr. M. Tohidi for her contribution to interpret laboratory tests.

\section{Authors' contributions}

F.R.T. was involved in the study conception and design, analysis and interpretation of data, manuscript drafting and critically revising. M.R. was involved in the analysis of data. F.M. contributed to manuscript drafting and final approval of the version. F.F. and N.H. contributed to data collection and manuscript drafting. F.A. contributed to critically revising the final approval of the version. The author(s) read and approved the final manuscript.

\section{Funding}

The study was funded by the Research Institute for Endocrine Sciences, Shahid Beheshti University of Medical Sciences, Tehran, Iran.

\section{Availability of data and materials}

The primary data for this study is available from the authors on direct request.

\section{Declarations}

\section{Ethics approval and consent to participate}

The study was approved by the Medical ethics committee of Shahid Beheshti University of Medical Sciences. All subjects signed a written informed consent.

\section{Consent for publication}

Not applicable.

\section{Competing interests}

The authors declare that they have no competing interests.

\section{Author details}

${ }^{1}$ Reproductive Endocrinology Research Center, Research Institute for Endocrine Sciences, Shahid Beheshti University of Medical Sciences, P.O. Box:19395-4763, 24 Parvaneh, Yaman Street, Velenjak, I.R 1985717413 Tehran, Iran. ${ }^{2}$ Endocrine Research Center, Research Institute for Endocrine Sciences, Shahid Beheshti University of Medical Sciences, Tehran, Iran.

Received: 8 November 2020 Accepted: 4 May 2021

Published online: 22 May 2021

\section{References}

1. Azziz R, Carmina E, Dewailly D, Diamanti-Kandarakis E, Escobar-Morreale HF, Futterweit W, Janssen OE, Legro RS, Norman RJ, Taylor AE, et al. The Androgen Excess and PCOS Society criteria for the polycystic ovary syndrome: the complete task force report. Fertil Steril. 2009;91:456-88.

2. Revised 2003 consensus. on diagnostic criteria and long-term health risks related to polycystic ovary syndrome (PCOS). Hum Reprod. 2004;19:41-7.

3. Dewailly D, Gronier H, Poncelet E, Robin G, Leroy M, Pigny P, Duhamel A, Catteau-Jonard S. Diagnosis of polycystic ovary syndrome (PCOS): revisiting the threshold values of follicle count on ultrasound and of the serum $\mathrm{AMH}$ level for the definition of polycystic ovaries. Hum Reprod. 2011;26:3123-9.

4. Sahmay S, Atakul N, Aydogan B, Aydin Y, Imamoglu M, Seyisoglu H. Elevated serum levels of anti-Mullerian hormone can be introduced as a new diagnostic marker for polycystic ovary syndrome. Acta Obstet Gynecol Scand. 2013;92:1369-74.
5. Teede H, Misso M, Tassone EC, Dewailly D, Ng EH, Azziz R, Norman RJ, Andersen M, Franks S, Hoeger K. Anti-Müllerian hormone in PCOS: a review informing international guidelines. Trends Endocrinol Metab. 2019;30(7): 467-78

6. Piltonen T, Morin-Papunen L, Koivunen R, Perheentupa A, Ruokonen A, Tapanainen JS. Serum anti-Mullerian hormone levels remain high until late reproductive age and decrease during metformin therapy in women with polycystic ovary syndrome. Hum Reprod. 2005;20:1820-6.

7. Dewailly D, Robin G, Peigne M, Decanter C, Pigny P, Catteau-Jonard S. Interactions between androgens, FSH, anti-Mullerian hormone and estradiol during folliculogenesis in the human normal and polycystic ovary. Hum Reprod Update. 2016;22:709-24.

8. Laven JS, Mulders AG, Visser JA, Themmen AP, De Jong FH, Fauser BC. AntiMullerian hormone serum concentrations in normoovulatory and anovulatory women of reproductive age. J Clin Endocrinol Metab. 2004;89:318-23.

9. Abbara A, Eng PC, Phylactou M, Clarke SA, Hunjan T, Roberts R, Vimalesvaran S, Christopoulos G, Islam R, Purugganan K, et al. Anti-Mullerian hormone $(\mathrm{AMH})$ in the Diagnosis of Menstrual Disturbance Due to Polycystic Ovarian Syndrome. Front Endocrinol (Lausanne). 2019;10:656.

10. Pigny $P$, Jonard $S$, Robert $Y$, Dewailly $D$. Serum anti-Mullerian hormone as a surrogate for antral follicle count for definition of the polycystic ovary syndrome. J Clin Endocrinol Metab. 2006;91:941-5.

11. Lauritsen MP, Bentzen JG, Pinborg A, Loft A, Forman JL, Thuesen LL, Cohen A, Hougaard DM, Nyboe Andersen A. The prevalence of polycystic ovary syndrome in a normal population according to the Rotterdam criteria versus revised criteria including anti-Mullerian hormone. Hum Reprod. 2014; 29:791-801.

12. Casadei L, Madrigale A, Puca F, Manicuti C, Emidi E, Piccione E, Dewailly D. The role of serum anti-Mullerian hormone $(\mathrm{AMH})$ in the hormonal diagnosis of polycystic ovary syndrome. Gynecol Endocrinol. 2013;29:545-50.

13. Tehrani FR, Solaymani-Dodaran M, Tohidi M, Gohari MR, Azizi F. Modeling age at menopause using serum concentration of anti-mullerian hormone. J Clin Endocrinol Metab. 2013;98:729-35.

14. Ramezani Tehrani F, Bidhendi Yarandi R, Solaymani-Dodaran M, Tohidi M, Firouzi F, Azizi F. Improving prediction of age at menopause using multiple anti-mullerian hormone measurements: the tehran lipid-glucose study. J Clin Endocrinol Metab. 2020;105:1-27.

15. Tehrani FR, Rashidi H, Azizi F. The prevalence of idiopathic hirsutism and polycystic ovary syndrome in the Tehran Lipid and Glucose Study. Reprod Biol Endocrinol. 2011;9:144

16. Tehrani FR, Simbar M, Tohidi M, Hosseinpanah F, Azizi F. The prevalence of polycystic ovary syndrome in a community sample of Iranian population: Iranian PCOS prevalence study. Reprod Biol Endocrinol. 2011;9:39.

17. Hastie TJ, Tibshirani RJ. Generalized additive models. CRC Press. London: Chapman and Hall; 1990.

18. Barrio I, Rodríguez-Álvarez M-X, Arostegui I: Categorization of Continuous Variables in a Logistic Regression Model Using the R Package CatPredi. 2015.

19. Vradi E, Jaki T, Vonk R, Brannath W. A Bayesian model to estimate the cutoff and the clinical utility of a biomarker assay. Stat Methods Med Res. 2019;28: 2538-56.

20. Carlin BP, Chib S. Bayesian model choice via Markov chain Monte Carlo methods. J Roy Stat Soc: Ser B (Methodol). 1995;57:473-84.

21. Smid SC, McNeish D, Miočević M, van de Schoot R. Bayesian versus frequentist estimation for structural equation models in small sample contexts: A systematic review. Structural Equation Modeling: A Multidisciplinary Journal. 2020;27:131-61.

22. McNeish D. On using Bayesian methods to address small sample problems. Structural Equation Modeling: A Multidisciplinary Journal. 2016;23:750-73.

23. Wang ET, Kao C-N, Shinkai K, Pasch L, Cedars Ml, Huddleston HG. Phenotypic comparison of Caucasian and Asian women with polycystic ovary syndrome: a cross-sectional study. Fertil Steril. 2013;100:214-8.

24. Api M, Badoglu B, Akca A, Api O, Gorgen H, Cetin A. Interobserver variability of modified Ferriman-Gallwey hirsutism score in a Turkish population. Arch Gynecol Obstet. 2009;279:473.

25. Streuli I, Fraisse T, Pillet C, Ibecheole V, Bischof P, De Ziegler D. Serum antimüllerian hormone levels remain stable throughout the menstrual cycle and after oral or vaginal administration of synthetic sex steroids. Fertil Steril. 2008:90:395-400.

26. Broer SL, Broekmans FJ, Laven JS, Fauser BC. Anti-Müllerian hormone: ovarian reserve testing and its potential clinical implications. Hum Reprod Update. 2014;20:688-701. 
27. Wiweko B, Maidarti M, Priangga MD, Shafira N, Fernando D, Sumapraja K, Natadisastra M, Hestiantoro A. Anti-mullerian hormone as a diagnostic and prognostic tool for PCOS patients. J Assist Reprod Genet. 2014;31:1311-6.

28. Homburg R, Ray A, Bhide P, Gudi A, Shah A, Timms P, Grayson K. The relationship of serum anti-Mullerian hormone with polycystic ovarian morphology and polycystic ovary syndrome: a prospective cohort study. Hum Reprod. 2013;28:1077-83.

29. Song DK, Oh J-Y, Lee H, Sung Y-A. Differentiation between polycystic ovary syndrome and polycystic ovarian morphology by means of an antiMüllerian hormone cutoff value. The Korean J Intern Med. 2017;32:690-8.

30. Oh SR, Choe SY, Cho YJ. Clinical application of serum anti-Müllerian hormone in women. Clin Exp Reprod Med. 2019;46:50.

31. Sova H, Unkila-Kallio L, Tiitinen A, Hippeläinen M, Perheentupa A, Tinkanen H, Puukka K, Bloigu R, Piltonen T, Tapanainen JS. Hormone profiling, including anti-Müllerian hormone (AMH), for the diagnosis of polycystic ovary syndrome (PCOS) and characterization of PCOS phenotypes. Gynecol Endocrinol. 2019;35:595-600.

32. Ahmad AK, Quinn M, Kao C-N, Greenwood E, Cedars MI, Huddleston HG Improved diagnostic performance for the diagnosis of polycystic ovary syndrome using age-stratified criteria. Fertil Steril. 2019;111:787-93. e782.

33. Mulders AG, Laven JS, Eijkemans MJ, de Jong FH, Themmen AP, Fauser BC Changes in anti-Mullerian hormone serum concentrations over time suggest delayed ovarian ageing in normogonadotrophic anovulatory infertility. Hum Reprod. 2004;19:2036-42.

34. de Kat AC, van der Schouw YT, Eijkemans MJ, Herber-Gast GC, Visser JA, Verschuren WM, Broekmans FJ. Back to the basics of ovarian aging: a population-based study on longitudinal anti-Mullerian hormone decline. BMC Med. 2016;14:151

35. Iliodromiti S, Kelsey TW, Anderson RA, Nelson SM. Can anti-Müllerian hormone predict the diagnosis of polycystic ovary syndrome? A systematic review and meta-analysis of extracted data. J Clin Endocrinol Metab. 2013; 98:3332-40.

36. Casadei L, Fanisio F, Sorge RP, Collamarini M, Piccolo E, Piccione E. The diagnosis of PCOS in young infertile women according to different diagnostic criteria: the role of serum anti-Müllerian hormone. Arch Gynecol Obstet. 2018;298:207-15.

37. Barrio I, Arostegui I, Quintana JM. Use of generalised additive models to categorise continuous variables in clinical prediction. BMC Med Res Methodol. 2013;13:83.

38. Quinn MM, Kao CN, Ahmad AK, Haisenleder DJ, Santoro N, Eisenberg E, Legro RS, Cedars MI, Huddleston HG, Network NNRM. Age-stratified thresholds of anti-Müllerian hormone improve prediction of polycystic ovary syndrome over a population-based threshold. Clin Endocrinol. 2017; 87:733-40.

39. Tola $H$, Abbas M, Alhassan EA, Shrif NE, Rida M. Assessment of the role of the anti-mullerian hormone, luteinizing hormone/follicle stimulating hormone ratio in the diagnosis of polycystic ovary syndrome in Sudanese women. Open Access Maced J Med Sci. 2018;6:1244.

40. Moslehi N, Shab-Bidar S, Ramezani Tehrani F, Mirmiran P, Azizi F. Is ovarian reserve associated with body mass index and obesity in reproductive aged women? A meta-analysis. Menopause. 2018;25:1046-55.

41. Rupp AA, Dey DK, Zumbo BD. To Bayes or not to Bayes, from whether to when: Applications of Bayesian methodology to modeling. Struct Equ Model. 2004;11:424-51

42. Kruschke JK, Aguinis $\mathrm{H}$, Joo H. The time has come: Bayesian methods for data analysis in the organizational sciences. Organizational Research Methods. 2012;15:722-52.

43. Amiri M, Tehrani FR, Behboudi-Gandevani S, Bidhendi-Yarandi R, Carmina E. Risk of hypertension in women with polycystic ovary syndrome: a systematic review, meta-analysis and meta-regression. Reprod Biol Endocrinol. 2020;18:1-15.

44. Ramezani Tehrani F, Amiri M, Behboudi-Gandevani S, Bidhendi-Yarandi R, Carmina E. Cardiovascular events among reproductive and menopausal age women with polycystic ovary syndrome: a systematic review and metaanalysis. Gynecol Endocrinol. 2020;36:12-23.

45. Behboudi-Gandevani S, Tehrani FR, Hosseinpanah F, Khalili D, Cheraghi L, Kazemijaliseh H, Azizi F. Cardiometabolic risks in polycystic ovary syndrome: long-term population-based follow-up study. Fertil Steril. 2018;110:1377-86.

46. Iwase A, Osuka S, Nakamura T, Kato N, Takikawa S, Goto M, Kikkawa F. Usefulness of the Ultrasensitive Anti-Mullerian Hormone Assay for Predicting True Ovarian Reserve. Reprod Sci. 2016;23:756-60.
47. de Kat AC, Broekmans FJM, van Westing AC, Lentjes E, Verschuren WMM, van der Schouw YT. A quantitative comparison of anti-Mullerian hormone measurement and its shifting boundaries between two assays. Maturitas. 2017;101:12-6.

\section{Publisher's Note}

Springer Nature remains neutral with regard to jurisdictional claims in published maps and institutional affiliations.
Ready to submit your research? Choose BMC and benefit from:

- fast, convenient online submission

- thorough peer review by experienced researchers in your field

- rapid publication on acceptance

- support for research data, including large and complex data types

- gold Open Access which fosters wider collaboration and increased citations

- maximum visibility for your research: over $100 \mathrm{M}$ website views per year

At BMC, research is always in progress.

Learn more biomedcentral.com/submissions 